\title{
Collisions of trapped molecules with slow beams
}

\author{
T. V. Tscherbul, $, 1,2,{ }^{1}$ Z. Pavlovic, ${ }^{2,3}$ H. R. Sadeghpour, ${ }^{2}$ R. Côté, ${ }^{3}$ and A. Dalgarno ${ }^{1,2}$ \\ ${ }^{1}$ Harvard-MIT Center for Ultracold Atoms, Cambridge, Massachusetts 02138, USA \\ ${ }^{2}$ Institute for Theoretical Atomic, Molecular, and Optical Physics, Harvard-Smithsonian Center for Astrophysics, \\ Cambridge, Massachusetts 02138, USA \\ ${ }^{3}$ Department of Physics, University of Connecticut, Storrs, Connecticut 06269, USA
}

(Received 12 February 2010; published 10 August 2010)

\begin{abstract}
We present a theoretical study of molecular-trap loss induced by collisions with slow atomic beams based on an explicit analysis of collision kinematics in the laboratory frame and a rigorous quantum description of atom-molecule scattering in external fields. The theory is applied to elucidate the effects of nonuniform magnetic and optical trapping fields on low-temperature collisions of $\mathrm{OH}\left(J=3 / 2, M_{J}=3 / 2, f\right)$ molecules with ${ }^{4} \mathrm{He}$ atoms. Our calculations quantify the extent to which both elastic and inelastic cross sections are suppressed by external trapping fields, clarify the role of small-angle scattering in trap loss, and may benefit future experiments on collisional cooling of molecules in electromagnetic traps. The calculated cross sections for trap loss in ${ }^{4} \mathrm{He}+\mathrm{OH}$ collisions are consistent with recent experimental observations at low beam energies [B. C. Sawyer et al., Phys. Rev. Lett. 101, 203203 (2008)], demonstrating the importance of including the effects of nonuniform trapping fields in theoretical simulations of cold collision experiments with trapped molecules and slow atomic beams.
\end{abstract}

DOI: 10.1103/PhysRevA.82.022704

PACS number(s): $34.50 . \mathrm{Cx}, 34.10 .+\mathrm{x}$

\section{INTRODUCTION}

The creation of dense ensembles of cold polar molecules has prompted avid interest in the study of field-controlled molecular collisions at temperatures below $1 \mathrm{~K}$ [1-3]. In addition to the electronic and hyperfine structure they share with atoms, molecules possess rovibrational energy levels and body-fixed electric dipole moments, which lead to complicated and interesting collision physics [1,3]. It is therefore not surprising that, while interactions of ultracold atoms are well understood both experimentally and theoretically [2], the field of cold molecular collisions is still in its infancy. Collisions involving molecular species can be influenced by chemical interactions [3], electric-field-induced avoided crossings [4], and long-range dipole-dipole interactions [5]. Studying molecular collisions at low temperatures in the presence of external fields is a promising route toward exploring these interactions and their effects on macroscopic properties of cold and ultracold molecular gases [1,3].

In addition to the fundamental interest, experimental studies of atom-molecule collisions at low temperatures are driven by the practical need to produce dense and cold ensembles of molecules via sympathetic cooling. Inelastic collisions, which result in heating rather than cooling, must be infrequent enough ( 1 inelastic collision per $>100$ elastic collisions) to allow for efficient sympathetic cooling [6]. Collisions between $\mathrm{He}$ atoms and many $\Sigma$-state open-shell molecules satisfy this requirement, enabling cryogenic cooling of $\mathrm{CaH}$, $\mathrm{CaF}, \mathrm{NH}$, and ND molecules and studies of their radiative properties [7] and interactions with $\mathrm{He}$ atoms at milli-Kelvin temperatures $[6,8,9]$. In contrast, the interactions of ${ }^{2} \Pi$ molecules such as $\mathrm{OH}$ with $\mathrm{He}$ atoms [10,11], $\mathrm{Rb}$ atoms [12], and each other [13] are strongly anisotropic, which leads to rapid collisional relaxation of low-field-seeking Zeeman

\footnotetext{
*tshcherb@cfa.harvard.edu
}

states $[10,12,13]$ and precludes evaporative cooling of the molecules in static electromagnetic traps.

Another powerful technique for studying molecular collisions is based on Stark deceleration of molecular beams [14-17]. With this technique, molecular collisions can be studied in several ways, including crossed-beam scattering [15], colliding packets in a molecular synchrotron [14], and monitoring the decay of trapped molecules following collision with a beam. The latter strategy was pursued by Sawyer et al. [17], who recently reported measurements of trap loss induced by collisions of magnetically trapped $\mathrm{OH}$ molecules with a supersonic beam of He atoms. We have recently addressed this experiment by performing rigorous calculations of elastic and inelastic cross cross sections for $\mathrm{He}+\mathrm{OH}$ collisions in superimposed electric and magnetic fields $[10,11]$.

Recent developments in quantum scattering methodology $[18,19]$ have enabled rigorous calculations of atommolecule [9-11] and molecule-molecule [20] collisions in the presence of uniform electromagnetic fields. However, the effects of nonuniform trapping fields were not taken into account in these calculations. A nonuniform trapping field can recapture collision products, causing the observed cross section for trap loss to be smaller than the total collision cross section [21]. Measurements of trap loss induced by collisions of $\mathrm{Rb}$ atoms with room-temperature $\mathrm{Ar}$ gas have shown that the recapture effect can be pronounced even at very small trap depths [21]. These observations suggest that the proper account of nonuniform trapping potentials might be essential for quantitative interpretation of collision experiments with trapped atoms or molecules.

In this work, we present a rigorous theoretical analysis of cold collision experiments with trapped molecules and slow atomic beams. In Sec. II A, we derive the expressions for beam-gas collision cross sections in external fields. We then consider the effects of trapping fields on collision dynamics by extending the model of Ref. [21] to account for inelastic energy transfer, the finite kinetic energy of the target gas, 
and state-dependent trapping potentials (Sec. II B). These improvements allow us to analyze the effects of elastic and inelastic collisions on trap-loss dynamics for a range of incident beam energies. In Sec. III, we apply our formulation to the experimentally relevant case of $\mathrm{He}+\mathrm{OH}$ collisions in a magnetic trap and analyze the dependence of elastic and inelastic cross sections on trap depth. Our results suggest that the effects of trapping fields should be taken into account in theoretical simulations of cold atom-molecule collisions. Finally, we discuss possible implications of our analysis for future experiments on sympathetic and evaporative cooling of molecules (Sec. IV).

\section{THEORY}

\section{A. Beam-gas collision cross sections}

Consider a gas of molecules moving with constant velocity $\mathbf{v}_{g}=v_{g} \hat{\mathbf{v}}_{g}$ colliding with a beam of atoms with forward velocity $\mathbf{v}_{b}=v_{b} \hat{\mathbf{v}}_{b}$ in the laboratory-fixed frame (LF). We choose the $z$ axis of the LF frame to be parallel to the direction of the external field, as shown in Fig. 1(a). In the beam-gas collision configuration, the vector $\mathbf{v}_{b}$ is well defined, and the orientation of $\mathbf{v}_{g}$ is determined by the velocity distribution of the target gas. Here we assume that $v_{g}$ is fixed and $\hat{\mathbf{v}}_{g}$ has a uniform distribution. The LF kinetic energy of gas molecules is $E_{g}=\frac{1}{2} m_{g} v_{g}^{2}$ and that of beam particles is $E_{b}=\frac{1}{2} m_{b} v_{b}^{2}$. The

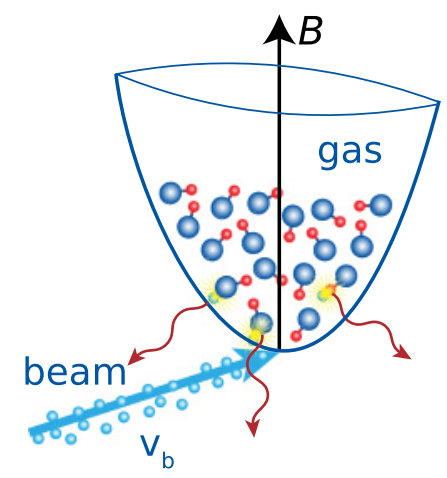

(a)

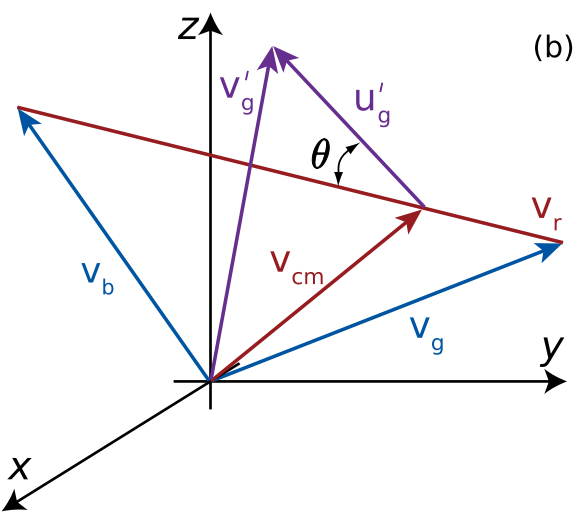

FIG. 1. (Color online) (a) Schematic illustration of the beam-gas collision experiment considered in this work. (b) Three-dimensional (3D) velocity vector diagram illustrating collision kinematics in the LF frame. All the vectors are defined in the text. relative velocity of collision partners is given by

$$
\mathbf{v}_{r}=\mathbf{v}_{g}-\mathbf{v}_{b},
$$

and the velocity of the center of mass (c.m.) is

$$
\mathbf{v}_{\text {c.m. }}=\left(m_{g} \mathbf{v}_{g}+m_{b} \mathbf{v}_{b}\right) / M,
$$

where $m_{g}$ and $m_{b}$ are masses of gas and beam particles and $M=m_{g}+m_{b}$ is the total mass. The vector $\mathbf{v}_{\mathrm{c} . \mathrm{m} \text {. defines }}$ the origin of the c.m. frame, in which the velocities of gas molecules are

$$
\begin{aligned}
& \mathbf{u}_{g}=\mathbf{v}_{g}-\mathbf{v}_{\text {c.m. }}, \\
& \mathbf{u}_{g}^{\prime}=\mathbf{v}_{g}^{\prime}-\mathbf{v}_{\text {c.m. }},
\end{aligned}
$$

where the primes serve to distinguish collision products. The relative velocity after collision is given by $\mathbf{v}_{r}^{\prime}=\mathbf{v}_{g}^{\prime}-\mathbf{v}_{b}^{\prime}=$ $\mathbf{u}_{g}^{\prime}-\mathbf{u}_{b}^{\prime}$, and it follows from conservation of momentum that

$$
\mathbf{u}_{g}=m_{b} \mathbf{v}_{r} / M
$$

and similarly $\mathbf{u}_{g}^{\prime}=m_{b} \mathbf{v}_{r}^{\prime} / M$. The c.m. collision energy is $E_{C}=\frac{1}{2} \mu v_{r}^{2}$, where $\mu=m_{b} m_{g} / M$ is the reduced mass for the collision.

In an idealized crossed-beam scattering experiment, $\hat{\mathbf{v}}_{r}$ is well defined and the flux of collision products in the direction specified by the vector $\hat{\mathbf{v}}_{r}^{\prime}$ can be measured, providing the differential cross section (DCS)

$$
\frac{d \sigma_{\gamma \rightarrow \gamma^{\prime}}}{d \Omega}\left(\hat{\mathbf{v}}_{r}, \hat{\mathbf{v}}_{r}^{\prime}, E_{C}\right)=\frac{1}{k_{\gamma}^{2}}\left|q_{\gamma \rightarrow \gamma^{\prime}}\left(\hat{\mathbf{v}}_{r}, \hat{\mathbf{v}}_{r}^{\prime} ; E_{C}\right)\right|^{2},
$$

where $k_{\gamma}^{2}=2 \mu\left(E-\epsilon_{\gamma}\right)=2 \mu E_{C}$ is the wave vector for the incident collision channel $\gamma$ with internal energy $\epsilon_{\gamma}, E$ is the total energy, and

$q_{\gamma \rightarrow \gamma^{\prime}}\left(\hat{\mathbf{v}}_{r}, \hat{\mathbf{v}}_{r}^{\prime}, E_{C}\right)=2 \pi \sum_{l, m_{l}} \sum_{l^{\prime}, m_{l}^{\prime}} \mathrm{i}^{l-l^{\prime}} Y_{l m_{l}}\left(\hat{\mathbf{v}}_{r}\right) Y_{l^{\prime} m_{l}^{\prime}}^{*}\left(\hat{\mathbf{v}}_{r}^{\prime}\right) T_{\gamma l m_{l} ; \gamma^{\prime} l^{\prime} m_{l}^{\prime}}$,

is the scattering amplitude [19,22]. In Eq. (6), $Y_{l m_{l}}$ are the spherical harmonics, and $\gamma$ and $\gamma^{\prime}$ refer to the internal (Stark and/or Zeeman) states of the molecule before and after the collision. The $T$-matrix elements $T_{\gamma l m_{l} ; \gamma^{\prime} l^{\prime} m_{l}^{\prime}}$ can be obtained by a numerical solution of the Schrödinger equation as described in Sec. II C.

The DCS defined by Eq. (5) is a function of four angles, which specify the orientation of vectors $\hat{\mathbf{v}}_{r}\left(\theta_{r}, \phi_{r}\right)$ and $\hat{\mathbf{v}}_{r}^{\prime}$ $\left(\theta_{r}^{\prime}, \phi_{r}^{\prime}\right)$ in the LF frame. According to the definition, the flux of scattered particles in state $\gamma^{\prime}$ into the element of the solid angle $d \Omega=d \hat{\mathbf{v}}_{r} d \hat{\mathbf{v}}_{r}^{\prime}$ is proportional, for a given collision energy $E_{C}$, to $\frac{d \sigma_{\gamma \rightarrow \gamma^{\prime}}}{d \Omega}\left(\hat{\mathbf{v}}_{r}, \hat{\mathbf{v}}_{r}^{\prime} ; E_{C}\right) d \Omega$ [23]. By integrating the DCS (5) over all possible directions of the outgoing collision flux and averaging over those of the initial flux, we obtain the integral cross section [19]

$$
\sigma_{\gamma \rightarrow \gamma^{\prime}}\left(E_{C}\right)=\frac{1}{4 \pi} \int d \hat{\mathbf{v}}_{r} \int d \hat{\mathbf{v}}_{r}^{\prime} \frac{d \sigma_{\gamma \rightarrow \gamma^{\prime}}}{d \Omega}\left(\hat{\mathbf{v}}_{r}, \hat{\mathbf{v}}_{r}^{\prime} ; E_{C}\right),
$$

which may be rewritten in terms of the $T$-matrix elements [19]

$$
\sigma_{\gamma \rightarrow \gamma^{\prime}}\left(E_{C}\right)=\frac{\pi}{k_{\gamma}^{2}} \sum_{l, m_{l}} \sum_{l^{\prime}, m_{l}^{\prime}}\left|T_{\gamma l m_{l} ; \gamma^{\prime} l^{\prime} m_{l}^{\prime}}\right|^{2}
$$


In a thermal gas, all directions of $\hat{\mathbf{v}}_{g}$ are equally probable, so the LF components of target gas velocity are not well defined $[24,25]$. This results in a distribution of relative collision velocities specified by Eq. (1), so beam-gas scattering cannot be described by the integral cross section evaluated at a fixed c.m. collision energy (8). A beam-gas collision cross section can be defined for a given incident beam orientation $\mathbf{v}_{b}$ and gas kinetic energy $E_{g}=\frac{1}{2} m_{g} v_{g}^{2}$ [24]

$$
\sigma_{\gamma \rightarrow \gamma^{\prime}}\left(\mathbf{v}_{b}, E_{g}\right)=\frac{1}{4 \pi} \int d \hat{\mathbf{v}}_{g} \int d \hat{\mathbf{v}}_{r}^{\prime} \frac{d \sigma_{\gamma \rightarrow \gamma^{\prime}}}{d \Omega}\left(\hat{\mathbf{v}}_{r}, \hat{\mathbf{v}}_{r}^{\prime} ; E_{C}\right) .
$$

Throughout this work, we assume that $E_{g}$ is fixed; the cross sections for the real thermal gas with temperature $T$ can be obtained by averaging Eq. (9) over a Maxwell-Boltzmann distribution of kinetic energies. It is instructive to compare Eq. (9) with the definition of the integral cross section (7). First, the integration in Eq. (9) is performed over target gas velocity $\hat{\mathbf{v}}_{g}$ rather than $\hat{\mathbf{v}}_{r}$. From Eq. (1), we have for the c.m. collision energy

$$
E_{C}\left(\mathbf{v}_{g}, \mathbf{v}_{b}\right)=\frac{1}{2} \mu\left[v_{g}^{2}+v_{b}^{2}-2 \mathbf{v}_{g} \cdot \mathbf{v}_{b}\right] .
$$

Therefore, the integration over $\hat{\mathbf{v}}_{g}$ in Eq. (9) with $\mathbf{v}_{b}$ held fixed is equivalent to averaging the scattering amplitude over a range of collision energies (10) and flux orientations $\hat{\mathbf{v}}_{r}$ and $\hat{\mathbf{v}}_{r}^{\prime}$. This is in contrast with the definition of the integral cross section (7), which is evaluated at a fixed collision energy, and the averaging is performed over $\hat{\mathbf{v}}_{r}$ and $\hat{\mathbf{v}}_{r}^{\prime}$ only. We return to this important point in Sec. III A.

In the limit $E_{g} \rightarrow 0$, Eq. (1) reduces to $\mathbf{v}_{r}=-\mathbf{v}_{b}$, so the averaging over $\hat{\mathbf{v}}_{g}$ in Eq. (9) becomes redundant, and we find

$$
\sigma_{\gamma \rightarrow \gamma^{\prime}}\left(\mathbf{v}_{b}, E_{g}=0\right)=\int d \hat{\mathbf{v}}_{r}^{\prime} \frac{d \sigma_{\gamma \rightarrow \gamma^{\prime}}}{d \Omega}\left(-\hat{\mathbf{v}}_{b}, \hat{\mathbf{v}}_{r}^{\prime} ; E_{C}\right) .
$$

This expression shows that the beam-gas collision cross section (9) has the same angular dependence as the DCS integrated over all directions of the outgoing collision flux. We emphasize that because the LF quantization axis $Z$ is defined by the external field, the cross sections defined by Eqs. (9) and (11) depend on the orientation of the beam velocity vector in the LF frame [26].

\section{B. Cross sections for trap loss}

If the target gas is confined in a trap, collision products may be recaptured by the trapping potential. To account for this effect, we assume that trap loss occurs when

$$
E_{g \gamma^{\prime}}>U_{\gamma^{\prime}},
$$

where $E_{g \gamma^{\prime}}=\frac{1}{2} m_{g} v_{g \gamma^{\prime}}^{2}$ is the LF kinetic energy of the gas molecule in the internal state $\gamma^{\prime}$ following the collision, and $U_{\gamma^{\prime}}$ is the trap depth. In this work, we consider both stateindependent and state-dependent trapping potentials, such as those of an optical dipole trap with a constant depth $U_{0}$ [27] and a permanent magnetic trap, for which [28]

$$
U_{\gamma^{\prime}}=\mu_{\gamma^{\prime}} B_{\max },
$$

where $\mu_{\gamma^{\prime}}$ is the magnetic moment of the molecule in state $\gamma^{\prime}$ and $B_{\max }$ is the magnetic field at the trap edge $[6,28]$. Magnetic trapping experiments are typically performed with molecules in maximally stretched low-field-seeking states [6]. In particular, the $\mathrm{OH}$ molecules were trapped in the state $\gamma=\left|J=3 / 2, M_{J}=3 / 2, f\right\rangle$, where $J$ is the total angular momentum of the molecule, $M_{J}$ is its projection on the magnetic-field axis, and $f$ is the inversion parity $[10,11]$. The state-dependent magnetic trapping potentials satisfy

$$
U_{\gamma^{\prime}}=\frac{M_{J}^{\prime}}{M_{J}} U_{0}
$$

where $U_{0}=\mu_{\gamma} B_{\max }$ is the maximum attainable trap depth (for $\left.M_{J}=3 / 2\right)$ and $M_{J}^{\prime}$ is the total angular momentum projection for the state $\gamma^{\prime}$. Equation (14) illustrates that the trapping potential felt by $\mathrm{OH}$ molecules in the $M_{J}^{\prime}=1 / 2$ state is three times smaller than for molecules in the $M_{J}^{\prime}=3 / 2$ state, and that molecules in $M_{J}^{\prime}<0$ states are antitrapped, that is, experience a repulsive force pushing them away from the center of the trap. This is in contrast with the optical dipole trapping potential, which is the same for all Zeeman states. As discussed in Sec. III, this difference has important consequences for the dependence of inelastic cross sections on trap depth.

The energy cutoff criterion (12) ensures that only collisions which impart enough kinetic energy to the trapped molecules result in trap loss. With this in mind, the cross section for trap loss may be written as [30]

$$
\begin{aligned}
& \sigma_{\gamma \rightarrow \gamma^{\prime}}^{\text {loss }}\left(\mathbf{v}_{b}, E_{g}, U_{0}\right) \\
& \quad=\frac{1}{4 \pi} \int d \hat{\mathbf{v}}_{g} \int d \hat{\mathbf{v}}_{r}^{\prime} \frac{d \sigma_{\gamma \rightarrow \gamma^{\prime}}}{d \Omega}\left(\hat{\mathbf{v}}_{r}, \hat{\mathbf{v}}_{r}^{\prime} ; E_{C}\right) h\left(E_{g \gamma^{\prime}}-U_{\gamma^{\prime}}\right),
\end{aligned}
$$

where $h(x)$ is the Heaviside step function, which serves to impose the condition (12). By comparing Eqs. (9) and (15), we find

$$
\lim _{U_{0} \rightarrow 0} \sigma_{\gamma \rightarrow \gamma^{\prime}}^{\text {loss }}\left(\mathbf{v}_{b}, E_{g}, U_{0}\right)=\sigma_{\gamma \rightarrow \gamma^{\prime}}\left(\mathbf{v}_{b}, E_{g}\right) .
$$

Thus, the cross section defined by Eq. (9) is a particular case of Eq. (15) in the limit of vanishing trap depth. By definition, the cross section for trap loss is always smaller than the total cross section for beam-gas collisions (9).

In order to evaluate Eq. (15), we need to know the LF kinetic energy of the trapped molecule after the collision $E_{g \gamma^{\prime}}=E_{g \gamma}+\Delta E_{g}$, where

$$
\Delta E_{g}=\frac{m_{g}}{2}\left[v_{g \gamma^{\prime}}^{2}-v_{g \gamma}^{2}\right] .
$$

This can be rewritten using Eq. (3) to yield

$$
\Delta E_{g}=\frac{m_{g}}{2}\left[u_{g \gamma^{\prime}}^{2}-u_{g \gamma}^{2}+2 \Delta \mathbf{u}_{g} \cdot \mathbf{v}_{\text {c.m. }} .,\right.
$$

where $\Delta \mathbf{u}_{g}=\mathbf{u}_{g \gamma^{\prime}}-\mathbf{u}_{g \gamma}$ is the collision-induced change in the c.m. velocity of the molecule. It follows from energy conservation that [29]

$$
\frac{1}{2} \mu v_{r \gamma}^{2}=\frac{1}{2} \mu v_{r \gamma^{\prime}}^{2}-\Delta \epsilon_{\gamma \gamma^{\prime}},
$$

where $\Delta \epsilon_{\gamma \gamma^{\prime}}=\epsilon_{\gamma}-\epsilon_{\gamma^{\prime}}$ is the energy difference between the initial and final collision channels. We note that for elastic collisions $\Delta \epsilon_{\gamma \gamma^{\prime}}=0$ and for the relaxation processes leading 
to trap loss $\Delta \epsilon_{\gamma \gamma^{\prime}}>0$. Making use of Eqs. (4) and (19) to simplify Eq. (18), we obtain

$$
\Delta E_{g}=m_{g}\left[\frac{m_{b}^{2}}{M^{2} \mu} \Delta \epsilon_{\gamma \gamma^{\prime}}+\Delta \mathbf{u}_{g} \cdot \mathbf{v}_{\mathrm{c} . \mathrm{m} .}\right] .
$$

The scalar product $\Delta \mathbf{u}_{g} \cdot \mathbf{v}_{\mathrm{c} . \mathrm{m}}$. can be evaluated from Cartesian components of the vectors $\Delta \mathbf{u}_{g}$ and $\mathbf{v}_{\mathrm{c} . \mathrm{m}}$. in the LF frame. Combining Eqs. (4) and (19), we find

$$
\begin{gathered}
\Delta u_{g x}=m_{b} v_{r \gamma}\left[r_{\gamma \gamma^{\prime}} \sin \theta_{r}^{\prime} \cos \phi_{r}^{\prime}-\sin \theta_{r} \cos \phi_{r}\right] / M, \\
\Delta u_{g y}=m_{b} v_{r \gamma}\left[r_{\gamma \gamma^{\prime}} \sin \theta_{r}^{\prime} \sin \phi_{r}^{\prime}-\sin \theta_{r} \sin \phi_{r}\right] / M, \\
\Delta u_{g z}=m_{b} v_{r \gamma}\left[r_{\gamma \gamma^{\prime}} \cos \theta_{r}^{\prime}-\cos \theta_{r}\right] / M,
\end{gathered}
$$

where

$$
r_{\gamma \gamma^{\prime}}=\frac{v_{r \gamma^{\prime}}}{v_{r \gamma}}=\left[1+\frac{\Delta \epsilon_{\gamma \gamma^{\prime}}}{E_{C}}\right]^{1 / 2}
$$

is the ratio of the relative velocities in the outgoing and incoming collision channels. The LF components of the vector $\mathbf{v}_{\text {c.m. }}$ can be assembled from those of $\mathbf{v}_{b}$ and $\mathbf{v}_{g}$ via Eq. (2). The angles $\theta_{r}^{\prime}\left(\theta_{r}\right)$ and $\phi_{r}^{\prime}\left(\phi_{r}\right)$ are the spherical polar angles of vectors $\hat{\mathbf{v}}_{r}^{\prime}\left(\hat{\mathbf{v}}_{r}\right)$ in the LF frame. Because the scattering amplitude (6) depends on the very same angles, Eq. (15) can be readily evaluated by numerical quadrature.

\section{Scattering calculations}

The cross sections for collisions of trapped $\mathrm{OH}$ molecules in the low-field-seeking state $|J=3 / 2, M=3 / 2, f\rangle$ with a beam of ${ }^{4} \mathrm{He}$ atoms are evaluated using Eq. (15). For a given beam orientation specified by the angles $\theta_{b}$ and $\phi_{b}$, a uniform grid over $\theta_{g}$ and $\phi_{g}$ is generated and the LF components of the vectors $\mathbf{v}_{r}$ and $\mathbf{v}_{\text {c.m. }}$ are evaluated using the definitions (1) and (2). The DCSs (5) are assembled from the real and imaginary parts of the scattering amplitude (6) calculated on four-dimensional grids over scattering angles $\left(\theta_{r}^{\prime}, \phi_{r}^{\prime}\right)$ and $\left(\theta_{r}, \phi_{r}\right)$ and collision energies (10). The LF energy of the gas molecule after the collision is computed using Eq. (17), and the criterion (12) is used to evaluate the integrand in Eq. (15). The $T$-matrix elements are obtained from rigorous quantum scattering calculations based on accurate $a b$ initio interaction potentials for $\mathrm{He}-\mathrm{OH}[10,11]$.

In the remainder of this article, we discuss how elastic and inelastic collisions contribute to trap loss. We define the contributions as

$$
p_{\text {inel }}=\sigma_{\text {inel }}^{\text {loss }} / \sigma_{\text {tot }}^{\text {loss }}
$$

and $p_{\mathrm{el}}=1-p_{\text {inel }}$, where

$$
\sigma_{\text {tot }}^{\text {loss }}=\sigma_{\mathrm{el}}^{\text {loss }}+\sigma_{\text {inel }}^{\text {loss }}
$$

is the total cross section for trap loss expressed via its elastic and inelastic counterparts

$$
\begin{gathered}
\sigma_{\mathrm{el}}^{\text {loss }}=\sigma_{\gamma \rightarrow \gamma}^{\text {loss }}, \\
\sigma_{\text {inel }}^{\text {loss }}=\sum_{\gamma^{\prime} \neq \gamma} \sigma_{\gamma \rightarrow \gamma^{\prime}}^{\text {loss }} .
\end{gathered}
$$

The $\mathrm{OH}$ molecules considered here are always in the maximally spin-stretched low-field-seeking Zeeman state $|\gamma\rangle=$ $|J=3 / 2, M=3 / 2, f\rangle$, so we omit the label $\gamma$ for brevity.
Unless stated otherwise, all calculations are performed for zero electric field and $B=0.93 \mathrm{~T}$, which is the average magnetic field $\bar{B}=3 B_{\max } / 2 \eta$ [28] experienced by the $\mathrm{OH}$ molecules trapped in the Zeeman state $\gamma=\left|J=3 / 2, M_{J}=3 / 2, f\right\rangle[31]$ in a 0.5 -K-deep magnetic trap, and $\eta=U_{0} / E_{g}$ is the reduced trap depth.

\section{RESULTS}

\section{A. Cross sections for beam-gas collisions versus collision energy and trap depth}

Figure 2 shows the cross sections for elastic scattering and inelastic relaxation in $\mathrm{He}+\mathrm{OH}$ collisions calculated as functions of the incident beam energy $E_{b}$ in the absence of a trapping field. To determine the range of c.m. collision energies sampled in beam-gas collisions, we rewrite Eq. (10) in the form

$$
E_{C}\left(\mathbf{v}_{g}, \mathbf{v}_{b}\right)=\mu\left[E_{g} / m_{g}+E_{b} / m_{b}-2\left(\frac{E_{g} E_{b}}{m_{g} m_{b}}\right)^{1 / 2} \hat{\mathbf{v}}_{g} \cdot \hat{\mathbf{v}}_{b}\right]
$$

so the minimum and maximum possible values of collision energy are

$$
E_{C \pm}=\mu\left[E_{g} / m_{g}+E_{b} / m_{b} \pm 2\left(\frac{E_{g} E_{b}}{m_{g} m_{b}}\right)^{1 / 2}\right]
$$
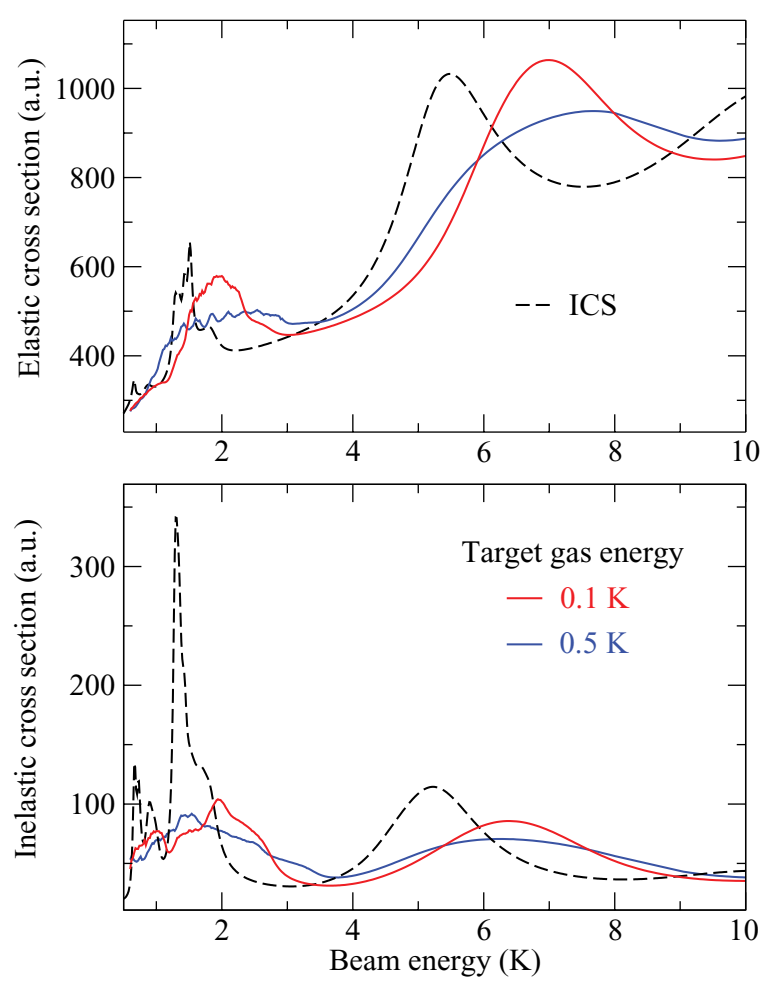

FIG. 2. (Color online) The cross sections for elastic scattering (upper panel) and inelastic relaxation (lower panel) in $\mathrm{He}+\mathrm{OH}$ collisions plotted vs beam energy $E_{b} . \theta_{b}=90^{\circ}, \phi_{b}=0^{\circ}, E_{g}=0.5 \mathrm{~K}$, and $B=0.93 \mathrm{~T}$. Also shown by dashed lines are the integral cross sections (8) as functions of $E_{C}$. 

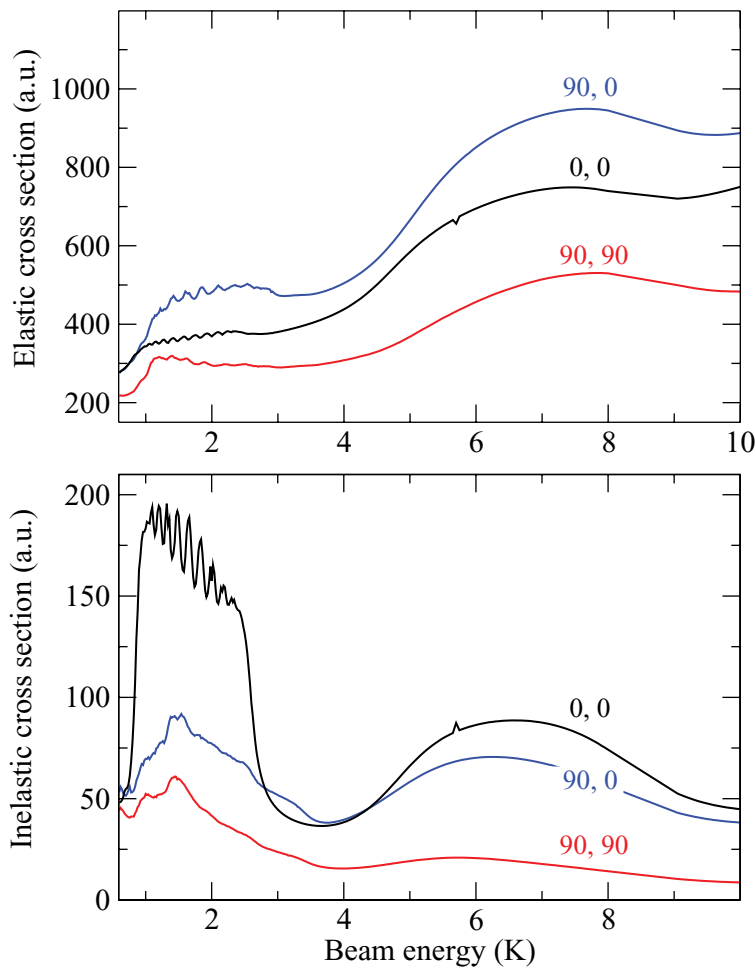

FIG. 3. (Color online) The cross sections for beam-gas collisions as functions of the beam energy for different orientations of the incoming beam in the LF frame. The curves are labeled by the beam angles $\theta_{b}, \phi_{b}$ (in degrees).

and the range of collision energies is

$$
\Delta E_{C}=E_{C+}-E_{C-}=4 \mu\left(\frac{E_{g} E_{b}}{m_{g} m_{b}}\right)^{1 / 2} .
$$

This result shows that the collision energy spread in beamgas experiments is proportional to the kinetic energies of the incident beam and target gas. As anticipated, collisions with warmer targets result in a wider spread in collision energies, leading to more pronounced averaging effects. Indeed, as shown in Fig. 2, the resonance structure apparent in the integral cross sections as functions of $E_{C}$ [11] is washed out in beam-gas collision cross sections plotted versus $E_{b}$.

Figure 3 shows the cross sections for $\mathrm{He}+\mathrm{OH}$ collisions as functions of the beam energy for different LF orientations of the incoming beam. The cross sections evaluated at different beam angles have similar shapes but can differ strongly in magnitude, especially at low beam energies approaching the target kinetic energy. In the low-energy regime, rotating the incident beam from parallel $\left(\theta_{b}=0^{\circ}\right)$ to perpendicular $\left(\theta_{b}=90^{\circ}\right)$ configuration leads to a four-fold decrease of the inelastic cross sections, while the elastic cross sections increase. The inelastic cross sections are also sensitive to the azimuthal angle $\phi_{b}$, increasing by a factor of two as $\phi_{b}$ is varied from $0^{\circ}$ to $90^{\circ}$. The dependence of the beam-gas cross sections on the beam angles $\theta_{b}$ and $\phi_{b}$ is due to the anisotropy of the scattering amplitude (6). In the stationary target limit $\left(E_{g} \rightarrow 0\right)$, this dependence mirrors that of the DCS integrated over all directions of the outgoing collision flux (11). At finite
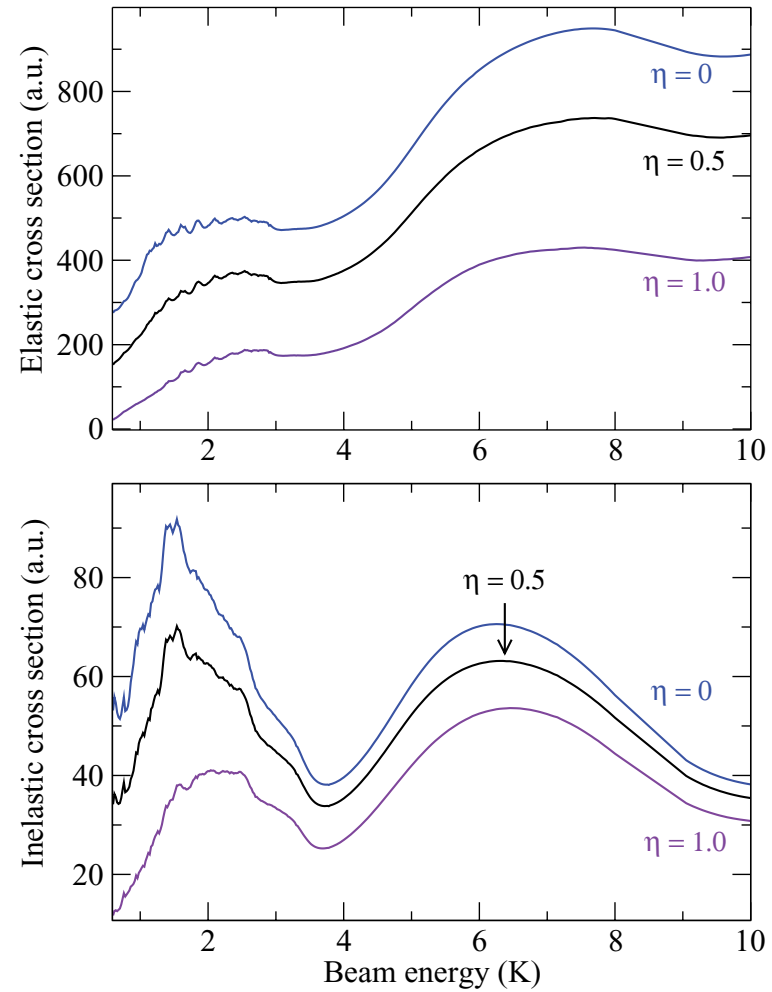

FIG. 4. (Color online) The cross sections for state-independent (optical) trap loss in $\mathrm{He}+\mathrm{OH}$ collisions as functions of the beam energy for different reduced trap depths $\eta$ indicated in the graph. $\theta_{b}=90^{\circ}, \phi_{b}=0^{\circ}, E_{g}=0.5 \mathrm{~K}$, and $B=0.93 \mathrm{~T}$.

$E_{g}$, the angular dependence of the beam-gas cross sections is modified by the averaging effects discussed in Sec. II A.

To examine the effects of trapping fields on beam-gas collisions, we first consider the case of the optical dipole trap with the same trap depth $U_{0}$ for all Zeeman states (13). Figure 4 shows the cross sections for trap loss in $\mathrm{He}+$ $\mathrm{OH}$ collisions as functions of the beam energy for different reduced trap depths $\eta=U_{0} / E_{g}$ [28]. As $\eta$ increases, fewer and fewer collisions satisfy the criterion (12), and both elastic and inelastic cross sections decrease. At low beam energies, the variation of cross sections with $E_{b}$ does not change dramatically with $\eta$. At $E_{b}>5 \mathrm{~K}$, the inelastic cross sections become less sensitive to $\eta$, whereas the elastic cross sections do not.

As shown in Fig. 5(a), both elastic and inelastic cross sections for trap loss decrease monotonically with increasing $\eta$. The elastic cross sections decrease more quickly, so trap loss at large $\eta$ occurs mainly via inelastic collisions. In the slow-beam regime $\left(E_{b} / E_{g} \sim 1\right)$, both elastic and inelastic cross sections are highly sensitive to trap depth. In particular, the cross sections for elastic scattering drop to zero and those for inelastic relaxation decrease by a factor of $\sim 17$ upon increasing $\eta$ from 0 to 3.3. We note that when $E_{b} / E_{g} \sim 1$, the energy release (17) is comparable to the trap depth, so it is possible to completely suppress collision-induced trap loss by choosing $\eta$ large enough so as to ensure that all scattered molecules will be recaptured by the trapping potential. 


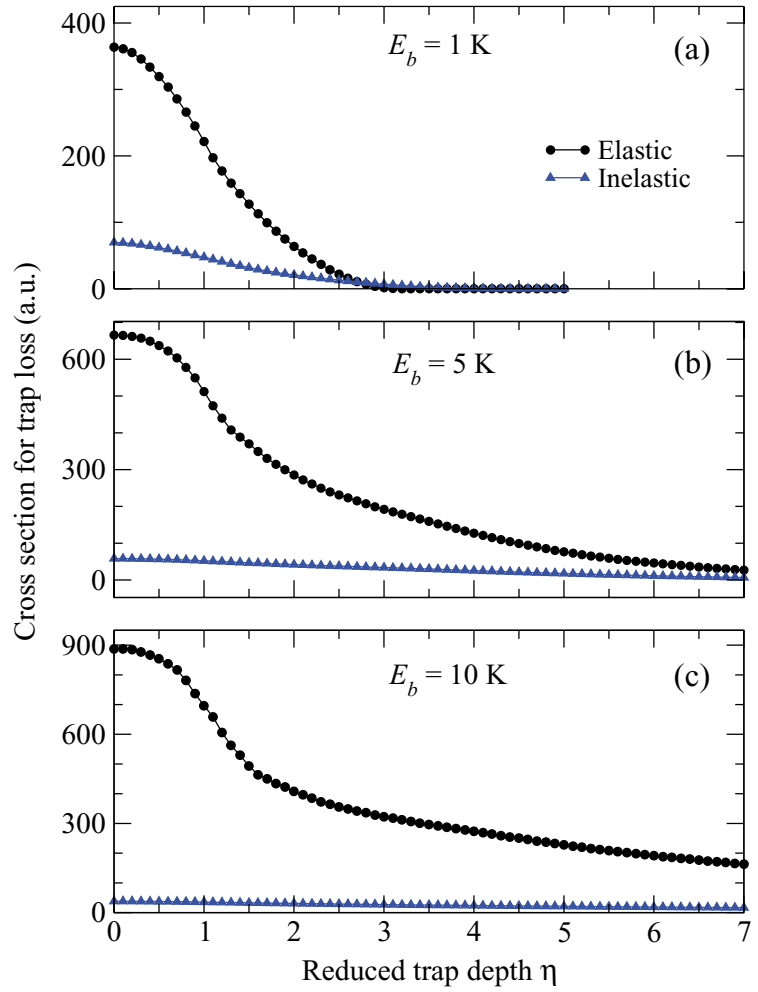

FIG. 5. (Color online) Elastic (circles) and inelastic (triangles) cross sections for state-independent (optical) trap loss in $\mathrm{He}+\mathrm{OH}$ collisions as functions of reduced trap depth $\eta$ for different incident beam energies: $E_{b}=1 \mathrm{~K}(\mathrm{a}), E_{b}=5 \mathrm{~K}$ (b), and $E_{b}=10 \mathrm{~K}$ (c). $\theta_{b}=90^{\circ}, \phi_{b}=0^{\circ}, E_{g}=0.5 \mathrm{~K}$, and $B=0.93 \mathrm{~T}$.

The situation is quite different for collisions of trapped molecules with fast beams. Figures 5 and 6 show that for $E_{b} / E_{g}>10$, the total cross section for trap loss is dominated by elastic collisions, which contribute at $>80 \%$ for $E_{b}=5 \mathrm{~K}$ and at $>90 \%$ for $E_{b}=10 \mathrm{~K}$. The inelastic contribution becomes smaller with increasing the beam energy or decreasing the trap depth. At $E_{b}=10 \mathrm{~K}$, the elastic and inelastic cross sections decrease by factors of 5.4 and 2.3 as $\eta$ is varied from 0 to 7 , showing much less sensitivity to $\eta$ compared to $E_{b}=5 \mathrm{~K}$, and especially to $E_{b}=1 \mathrm{~K}$. These results corroborate our previous conclusions [11] that magnetically trapped $\mathrm{OH}$ molecules colliding with fast $\mathrm{He}$ atoms from a supersonic beam [17] are likely to be lost via elastic collisions.

To clarify the dependence of the cross sections on trap depth, we use a simplified expression for the energy transfer (20) valid in the limit $E_{g} \rightarrow 0$ :

$$
\Delta E_{g}=\frac{2 \mu E_{C}}{m_{g}}(1-\cos \theta)+\frac{m_{g} m_{b}^{2}}{M^{2} \mu} \Delta \epsilon_{\gamma \gamma^{\prime}},
$$

where $\theta$ is the c.m. scattering angle between the vectors $\mathbf{v}_{r}$ and $\mathbf{u}_{g}^{\prime}$ (or $\mathbf{v}_{r}^{\prime}$ ) shown in Fig. 1. The first term on the right-hand side of Eq. (29) accounts for elastic collisions and has the same form as Eq. (1) of Ref. [21]. Given Eq. (29), the condition for trap loss (12) becomes

$$
\Delta E_{g}=\frac{m_{b}}{M}\left[2 E_{C}(1-\cos \theta)+\Delta \epsilon_{\gamma \gamma^{\prime}}\right]>U_{0} .
$$

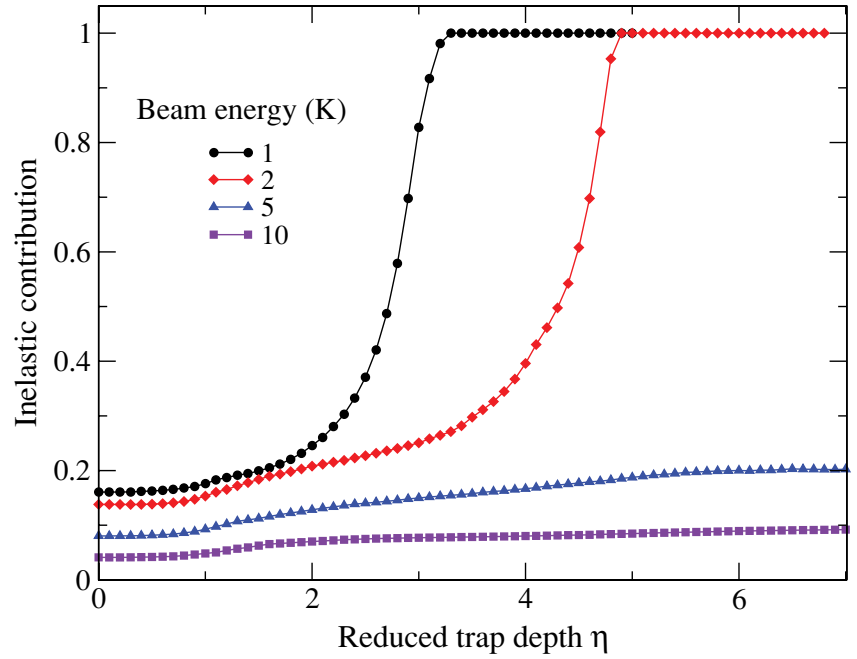

FIG. 6. (Color online) The inelastic contribution $p_{\text {inel }}$ to the total cross section for state-independent (optical) trap loss (23) plotted vs $\eta$ for different beam energies $E_{b}: 1 \mathrm{~K}$ (circles), $2 \mathrm{~K}$ (diamonds), $5 \mathrm{~K}$ (triangles), and $10 \mathrm{~K}$ (squares). $\theta_{b}=90^{\circ}, \phi_{b}=0^{\circ}, E_{g}=0.5 \mathrm{~K}$, and $B=0.93 \mathrm{~T}$.

Solving this inequality for $\theta$ and combining the result with the definition (15), we find

$$
\sigma_{\gamma \rightarrow \gamma^{\prime}}^{\text {loss }}=\int_{\theta_{\min }}^{\pi} \sin \theta d \theta \int_{0}^{2 \pi} d \phi \frac{d \sigma_{\gamma \rightarrow \gamma^{\prime}}}{d \Omega}\left(\theta, \phi ; E_{C}\right),
$$

where

$$
\theta_{\min }=\arccos \left(1-\frac{M U_{0} / m_{b}-\Delta \epsilon_{\gamma \gamma^{\prime}}}{2 E_{C}}\right)
$$

is the cutoff angle [21]. We note that the DCS in Eq. (31) is expressed through the c.m. scattering angles $\theta$ and $\phi$ and not the initial and final flux orientation angles as before. Equation (32) establishes that collisions which lead to smallangle scattering $\theta \in\left[0, \theta_{\min }\right]$ do not contribute to trap loss in the limit of large beam energy $\left(E_{b} / E_{g} \gg 1\right)$. Because $\theta_{\min }$ increases with $U_{0}$, the "forbidden" range of scattering angles widens with increasing trap depth, leading the cross sections for trap loss to decrease monotonically with $\eta$ [21].

As follows from Eqs. (31) and (32), the variation of the cross sections with trap depth is determined by the behavior of the DCS at small scattering angles. Figure 7 shows that the elastic DCS for $\mathrm{He}+\mathrm{OH}$ are strongly peaked in the forward direction $\left(\theta=0^{\circ}\right)$. This makes the elastic cross sections defined by Eq. (31) very sensitive to the cutoff parameter $\theta_{\max }$ and explains their rapid decline with $\eta$ shown in Fig. 5(c). In contrast, the DCSs for inelastic collisions do not exhibit any dramatic variations near $\theta=0^{\circ}$. Because Eq. (32) contains a constant term proportional to the energy release $\Delta \epsilon_{\gamma \gamma^{\prime}}$, the cutoff angle $\theta_{\min }$ for relaxation collisions $\left(\Delta \epsilon_{\gamma \gamma^{\prime}}>0\right.$ ) is smaller than for elastic collisions. For these reasons, the variation of the inelastic contribution to trap loss with $\eta$ is not as pronounced, as illustrated in Fig. 5(c).

In a permanent magnetic trap, inelastic collision products not only have more translational energy but also experience a 


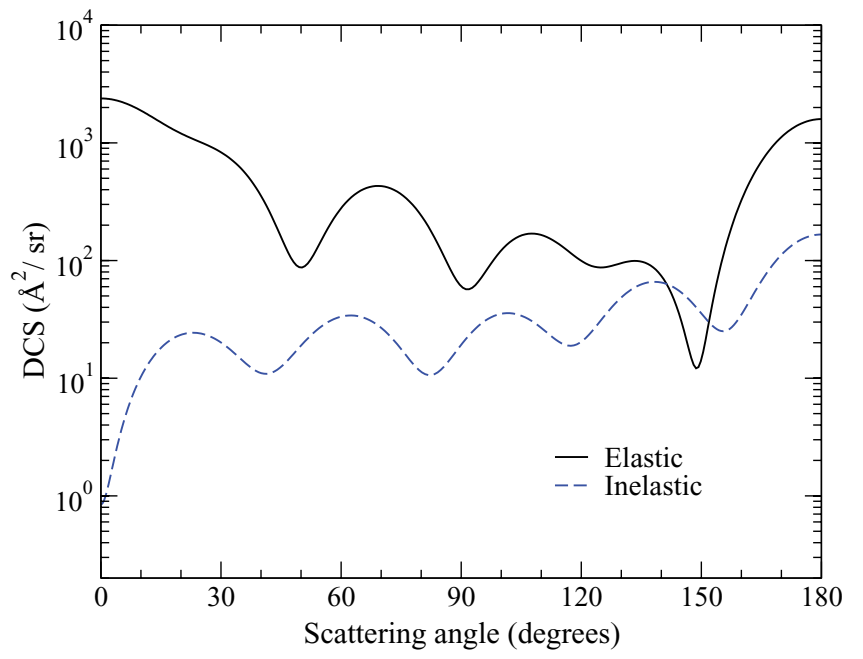

FIG. 7. (Color online) Elastic (full line) and inelastic (dashed line) DCSs for $\mathrm{He}+\mathrm{OH}$ as functions of $\theta_{r}^{\prime}$ for $E_{C}=3.0 \mathrm{~cm}^{-1}$, $B=0.93 \mathrm{~T}$, and $\phi_{r}^{\prime}=0^{\circ}$. The initial relative velocity vector is parallel to the LF quantization axis $\left(\theta_{r}=0^{\circ}, \phi_{r}=0^{\circ}\right)$.

reduced trapping potential. To account for this, we calculated the state-to-state inelastic cross sections with the trap depth parameter adjusted individually for each final state $M_{J}^{\prime}$ (14) and summed the results over $M_{J}^{\prime}$ to obtain the total cross sections for magnetic trap loss (25). Figure 8 shows that the

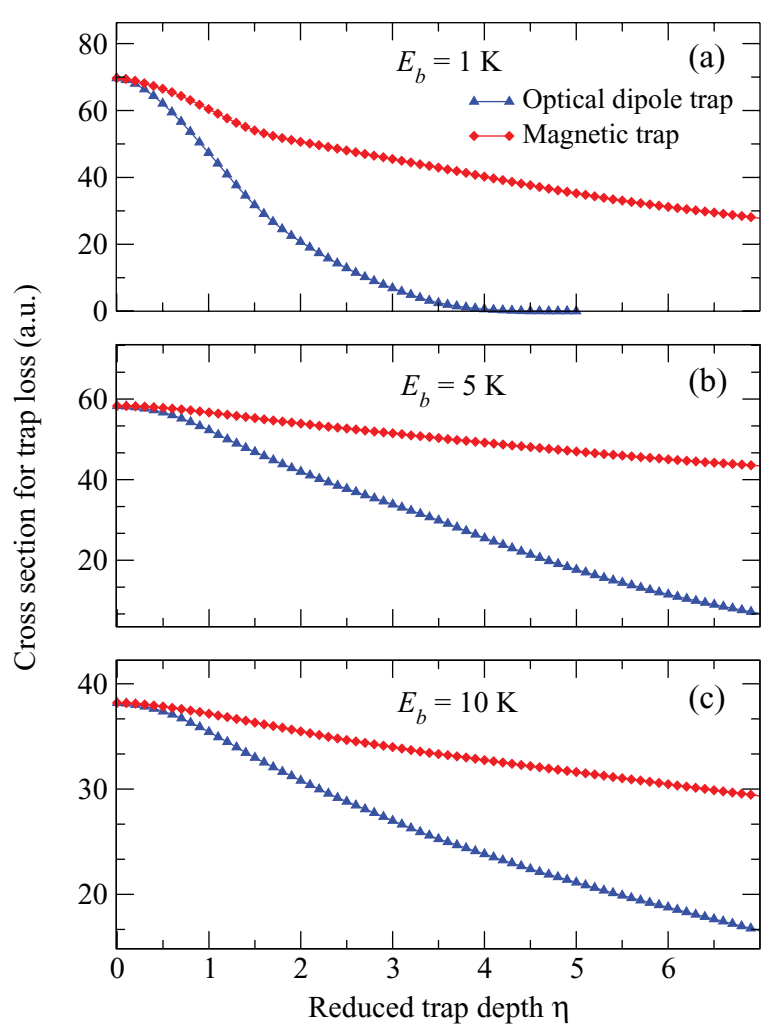

FIG. 8. (Color online) Inelastic cross sections for collisioninduced trap loss as functions of $\eta$ for different incident beam energies: $E_{b}=1 \mathrm{~K}(\mathrm{a}), E_{b}=5 \mathrm{~K}(\mathrm{~b})$, and $E_{b}=10 \mathrm{~K}$ (c). Triangles represent state-independent (optical) trap, and diamonds representstate-dependent (magnetic) trap. inelastic cross sections evaluated for a permanent magnetic trap are less sensitive to trap depth than those for an optical dipole trap. To explain this observation, we note that trapped $\mathrm{OH}\left(J=3 / 2, M_{J}=3 / 2, \epsilon=f\right)$ molecules can relax into three channels $\left|J^{\prime}, M_{J}^{\prime}, \epsilon^{\prime}\right\rangle$ of the same parity $\left(J^{\prime}=3 / 2\right.$, $\epsilon^{\prime}=f, M_{J}^{\prime}= \pm 1 / 2$ or $\left.-3 / 2\right)$ and four channels of the opposite parity $\left(J^{\prime}=3 / 2, \epsilon^{\prime}=e, M_{J}= \pm 3 / 2\right.$ or $\left.\pm 1 / 2\right)$ as the incident collision channel $[10,11]$. Of these seven relaxation channels, one $\left(M_{J}^{\prime}=3 / 2\right)$ feels the same trapping potential as the incident channel, the other two $\left(M_{J}^{\prime}=1 / 2\right)$ feel a potential which is three times weaker, and the remaining four are antitrapped. Since $U_{\gamma^{\prime}}<0$ for the antitrapped states, trap loss via inelastic transitions to these states is independent of $\eta$, which has the effect of weakening the dependence of the total inelastic cross sections (25) on trap depth.

\section{B. Comparison with experiment}

Sawyer et al. have recently measured the cross sections for trap loss in collisions of magnetically trapped $\mathrm{OH}(\mathrm{J}=$ $\left.3 / 2, M_{J}=3 / 2, f\right)$ with a slow beam of ${ }^{4} \mathrm{He}$ atoms [17] as functions of the beam kinetic energy in the interval $E_{b}=60-220 \mathrm{~cm}^{-1}$. They observed a shoulderlike feature near $E_{b} \sim 100 \mathrm{~cm}^{-1}$, which they tentatively attributed to a quantum mechanical threshold effect associated with the opening of the $J=5 / 2$ rotationally excited state of $\mathrm{OH}$ [17]. The experimental data are reproduced in Fig. 9.

To interpret the experimental observations, we carried out rigorous quantum scattering calculations based on the same set of $a b$ initio interaction potentials [32] as used in the present work. The calculations [11] demonstrated that the total cross section for $\mathrm{He}+\mathrm{OH}$ is dominated by the elastic cross section, which is a smoothly decreasing function of collision energy.

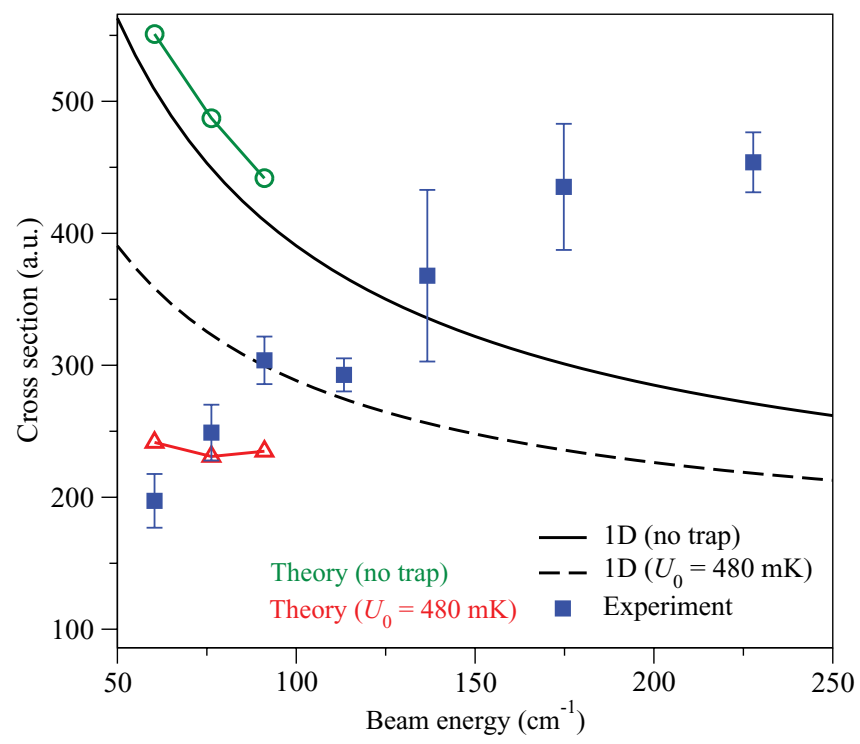

FIG. 9. (Color online) Total cross sections for trap loss (24) calculated for zero trap depth (circles), $U_{0}=480 \mathrm{mK}$ (triangles), 1D model for zero trap depth (full line), and the 1D model based on Eq. (31) (dashed line). The beam orientation angles are $\theta_{b}=90^{\circ}$ and $\phi_{b}=0^{\circ}$. The experimental results from Ref. [17] are shown as squares with error bars. 
As a result, no signature of the experimentally observed feature at $E_{b} \sim 100 \mathrm{~cm}^{-1}$ was seen in these calculations [11]. While imperfections in the $\mathrm{He}-\mathrm{OH}$ interaction potentials [32] could not account for the observed discrepancy [11], our calculations did not include the effects of nonuniform trapping fields, which might be responsible for the reduction of the observed cross sections.

To elucidate the effects of trapping fields on the dynamics of collision-induced trap loss, we evaluated the cross sections (15) using the same values of trapped $\mathrm{OH}$ kinetic energy $\left(E_{g}=70 \mathrm{mK}\right)$, average magnetic field $(\bar{B}=0.13 \mathrm{~T})$, and trap depth $\left(U_{0}=480 \mathrm{~K}\right)$ as in the experiments of Sawyer et al. [17]. For each $E_{b}$, the $T$-matrix elements were evaluated within the collision energy range specified by Eq. (28) with a grid step size of $0.01 \mathrm{~cm}^{-1}$ and used to assemble the cross sections for trap loss via Eq. (15). The integral in Eq. (15) was evaluated with fifty-five Gauss-Legendre quadrature points in $\theta_{r}$ and $\theta_{r}^{\prime}$ and sixty Gauss-Legendre quadrature points in $\phi_{r}$ and $\phi_{r}^{\prime}$. Such dense angular grids were required due to the highly oscillatory structure of the DCS at collision energies above $60 \mathrm{~cm}^{-1}$. For this reason, we were able to obtain converged cross sections only at the three lowest beam energies probed in the experiment [17].

Figure 9 compares the calculated and measured [17] total cross sections for trap loss in $\mathrm{He}+\mathrm{OH}$ collisions. The cross sections evaluated for $U_{0}=480 \mathrm{mK}$ are in better agreement with experimental results than those evaluated for zero trap depth [11], suggesting that nonuniform trapping fields have a large effect on the measured trap-loss dynamics [17]. Table I shows that the elastic component of the total cross section is most sensitive to trap depth and that the inelastic cross sections are almost unaffected by the trapping field. These observations are consistent with the tendencies discussed previously for lower beam energies (Figs. 5 and 7).

The cross sections for beam-gas collisions evaluated at zero trap depth (shown in Fig. 9 by circles) are similar to their elastic counterparts. The elastic cross sections are well described by a simple one-dimensional (1D) model that ignores the anisotropy of the $\mathrm{He}-\mathrm{OH}$ interaction potentials [11]. In contrast, the agreement between the cross sections evaluated for $U_{0}=480 \mathrm{mK}$ using Eq. (31) [21] and the accurate quantum results is poor at the lowest beam energy but improves at higher energies. This observation might reflect the inadequacy of the stationary target approximation [21] in the limit of small beam energies.

TABLE I. Total, elastic, and inelastic cross sections for beam-gas collisions calculated at the three incident beam energies probed in the experiment [17]. The cross sections are given in atomic units for $U_{0}=0$ and $U_{0}=480 \mathrm{mK}$ (in parentheses).

\begin{tabular}{lccc}
\hline \hline Beam energy $\left(\mathrm{cm}^{-1}\right)$ & Elastic & Inelastic & Total \\
\hline 60.38 & 543.3 & 7.7 & 551.0 \\
& $(234.4)$ & $(7.2)$ & $(241.5)$ \\
76.27 & 480.7 & 6.5 & 487.2 \\
& $(224.8)$ & $(6.1)$ & $(230.9)$ \\
91.10 & 435.8 & 5.9 & 441.7 \\
& $(229.3)$ & $(5.5)$ & $(234.9)$ \\
\hline \hline
\end{tabular}

\section{CONCLUSIONS}

We have presented a theoretical analysis of atom-molecule collisions in the presence of nonuniform trapping fields. The approach combines the energy cutoff criterion (12) for permanent magnetic and optical dipole traps [21] with the rigorous quantum treatment of atom-molecule collisions in external fields [19]. Our study shows that collisions of trapped $\mathrm{OH}$ molecules with slow beams of $\mathrm{He}$ atoms are sensitive to trap depth, the kinetic energy of the incident beam, and its orientation with respect to the quantization axis defined by the external field. The c.m. collision energy is not well defined for beam-gas collisions, and the observed cross sections are averages over a range of collision energies specified by the kinetic energies of the incoming beam and target gas (Sec. III).

The cross sections for trap loss can be evaluated from the DCSs by using the energy cutoff criterion (12), which states that only those collisions which impart enough kinetic energy to the trapped molecules result in trap loss. The energy transfer can be evaluated in terms of LF Cartesian components of the initial and final relative velocity vectors (21). In the fast beam limit $\left(E_{b} / E_{g} \gg 1\right)$, the LF energy transfer vanishes as the scattering angle approaches zero (Sec. III). As a result, elastic collisions which result in forward-scattered products $\left(\theta \approx 0^{\circ}\right)$ do not contribute to trap loss. Because the DCS for elastic scattering is typically strongly peaked at $\theta=0^{\circ}$ (Fig. 7), the elastic cross sections for trap loss are responsive even to small variations of the trap depth [Fig. 5(c)]. In contrast, the inelastic DCSs do not exhibit any dramatic variations in the vicinity of $\theta=0$, so the contribution to trap loss due to inelastic collisions is less sensitive to trap depth [Figs. 5(c) and 7]. These considerations do not apply when the kinetic energy of the incident beam is comparable to that of the target gas. In the slow beam regime $\left(E_{b} / E_{g} \sim 1\right)$, both elastic and inelastic cross sections are sensitive to trap depth, which makes it possible to suppress collisional trap loss by increasing $\eta$ [Figs. 5(a) and 6].

The dynamics of collision-induced trap loss is sensitive to the nature of the trapping potential. Our calculations show that state-independent (optical) trapping potentials lead to suppressed inelastic losses at large trap depths (Fig. 5), whereas inelastic collisions of molecules in state-dependent (magnetic) traps are less sensitive to trap depth (Fig. 8). Inelastic transitions release energy into the relative motion of collision partners and change their internal states, thereby weakening magnetic confinement. While collision-induced loss from optical dipole traps occurs via the energy release mechanism, both mechanisms are important for magnetic traps (Sec. III).

Our analysis suggests that external trapping fields modify the dynamics of molecular collisions at low temperatures and result in a reduction of the cross sections for trap loss. We find that quantum calculations of trap-loss dynamics are in better agreement with experimental results [17] when the effects of trapping fields are taken into account. We conclude that it is essential to include such effects in theoretical simulations of beam-trap collision experiments even when the incident beam energy is very high compared to that of the target gas $\left(E_{b} / E_{g} \approx\right.$ 860 for the lowest $E_{b}$ studied in the experiment [17]). 
In this work, we have assumed that the target gas velocity vector $v_{b}$ has a fixed length and points in arbitrary direction, which gives the cross section for collision-induced trap loss (15) as a function of $E_{g}$ and $E_{b}$. This cross section can be averaged over appropriately chosen distributions of $E_{g}$ and $E_{b}$ (such as the Maxwell-Boltzmann distribution) to yield the experimentally measurable trap loss rates.

Our findings may benefit future collision experiments with cold molecular gases confined by external electromagnetic fields. The techniques of evaporative and sympathetic cooling of molecular ensembles are based on collisional thermalization of trapped molecules at variable trap depths. Knowledge of collisional loss rates as functions of $\eta$ is critical to the success of evaporative cooling of molecules, and the present approach will help to optimize the ongoing experiments on evaporative cooling of molecular ensembles to quantum degeneracy. In particular, it will be possible to theoretically predict various undesirable effects such as stalled evaporation due to large inelastic relaxation rates [28].

The trap-loss rates inferred from cryogenic cell experiments are integral characteristics which contain contributions from a number of collisional mechanisms, including evaporation over the trap edge due to elastic collisions and inelastic relaxation [28]. The rates at which these processes occur depend on temperature and trap depth, which brings about a source of uncertainty to be properly addressed in experimental data analysis. One way to solve this problem is to perform collision experiments in very deep traps $(\eta>10)$, which ensures that elastic collisions do not contribute to trap loss. The formalism outlined in Sec. II allows for explicit calculations of collisional trap loss rates for arbitrary $\eta$, which might prove advantageous for cold collision experiments with weakly magnetic atoms and molecules [33], where the regime $\eta>10$ is difficult to achieve experimentally.

\section{ACKNOWLEDGMENTS}

We thank Colin Connolly, Vasili Kharchenko, Bas van de Meerakker, and Kirk Madison for valuable discussions and helpful comments on the manuscript. This work was supported by the Chemical Science, Geoscience, and Bioscience Division of the Office of Basic Energy Science, Office of Science, US Department of Energy, and NSF grants to the Harvard-MIT Center for Ultracold Atoms and the Institute for Theoretical Atomic, Molecular, and Optical Physics at Harvard University and Smithsonian Astrophysical Observatory.
[1] L. D. Carr, D. DeMille, R. V. Krems, and J. Ye, New J. Phys. 11, 055049 (2009).

[2] J. Weiner, V. S. Bagnato, S. Zilio, and P. S. Julienne, Rev. Mod. Phys. 71, 1 (1999); T. Kohler, K. Goral, and P. S. Julienne, ibid. 78, 1311 (2006); C. Chin, R. Grimm, P. Julienne, and E. Tiesinga, ibid. 82, 1225 (2010).

[3] R. V. Krems, Phys. Chem. Chem. Phys. 10, 4079 (2008).

[4] T. V. Tscherbul and R. V. Krems, Phys. Rev. Lett. 97, 083201 (2006).

[5] A. V. Avdeenkov and J. L. Bohn, Phys. Rev. Lett. 90, 043006 (2003); A. V. Avdeenkov, D. C. E. Bortolotti, and J. L. Bohn, Phys. Rev. A 69, 012710 (2004).

[6] J. M. Doyle, B. Friedrich, J. Kim, and D. Patterson, Phys. Rev. A 52, R2515 (1995).

[7] W. C. Campbell, G. C. Groenenboom, H. I. Lu, E. Tsikata, and J. M. Doyle, Phys. Rev. Lett. 100, 083003 (2008).

[8] J. D. Weinstein, R. deCarvalho, T. Guillet, B. Friedrich, and J. M. Doyle, Nature (London) 395, 148 (1998).

[9] W. C. Campbell, T. V. Tscherbul, H. I. Lu, E. Tsikata, R. V. Krems, and J. M. Doyle, Phys. Rev. Lett. 102, 013003 (2009).

[10] T. V. Tscherbul, G. Groenenboom, R. V. Krems, and A. Dalgarno, Faraday Discuss. 142, 127 (2009).

[11] Z. Pavlovic, T. V. Tscherbul, H. R. Sadeghpour, G. C. Groenenboom, and A. Dalgarno, J. Phys. Chem. A 113, 14670 (2009).

[12] M. Lara, J. L. Bohn, D. Potter, P. Soldán, and J. M. Hutson, Phys. Rev. Lett. 97, 183201 (2006).

[13] A. V. Avdeenkov and J. L. Bohn, Phys. Rev. A 66, 052718 (2002).
[14] C. E. Heiner, D. Carty, G. Meijer, and H. L. Bethlem, Nat. Phys. 3, 115 (2007).

[15] J. J. Gilijamse, S. Hoekstra, S. Y. T. van de Meerakker, G. C. Groenenboom, and G. Meijer, Science 313, 1617 (2006).

[16] S. Y. T. van de Meerakker and G. Meijer, Faraday Discuss. 142, 113 (2009).

[17] B. C. Sawyer, B. K. Stuhl, D. Wang, M. Yeo, and J. Ye, Phys. Rev. Lett. 101, 203203 (2008).

[18] A. Volpi and J. L. Bohn, Phys. Rev. A 65, 052712 (2002).

[19] R. V. Krems and A. Dalgarno, J. Chem. Phys. 120, 2296 (2004).

[20] T. V. Tscherbul, Y. V. Suleimanov, V. Aquilanti, and R. V. Krems, New J. Phys. 11, 055021 (2009).

[21] D. E. Fagnan, J. Wang, C. Zhu, P. Djuricanin, B. G. Klappauf, J. L. Booth, and K. W. Madison, Phys. Rev. A 80, 022712 (2009).

[22] A. M. Arthurs and A. Dalgarno, Proc. R. Soc. London A 256, 540 (1960); M. D. Rowe and A. J. McCaffery, Chem. Phys. 43, 35 (1979).

[23] G. G. Balint-Kurti, in International Review of Science, Series II, Vol. 1, edited by A. D. Buckingham and C. A. Coulson (Butterworths, London, 1975), p. 286.

[24] M. H. Alexander, P. J. Dagdigian, and A. E. DePristo, J. Chem. Phys. 66, 59 (1977).

[25] P. J. Dagdigian, H. W. Cruse, and R. N. Zare, J. Chem. Phys. 62, 1824 (1975); R. A. Gottscho, R. W. Field, R. Bacis, and S. J. Silvers, ibid. 73, 599 (1980); J. A. Kettleborough and K. G. McKendrick, J. Phys. Chem. 95, 8255 (1991).

[26] T. T. Warnock and R. B. Bernstein, J. Chem. Phys. 49, 1878 (1968)

[27] J. D. Miller, R. A. Cline, and D. J. Heinzen, Phys. Rev. A 47, R4567 (1993). 
[28] S. V. Nguyen, R. deCarvalho, and J. M. Doyle, Phys. Rev. A 75, 062706 (2007).

[29] T. V. Tscherbul, G. Barinovs, J. Kłos, and R. V. Krems, Phys. Rev. A 78, 022705 (2008).

[30] The absolute magnitudes of the vectors $\mathbf{v}_{r}, \mathbf{v}_{r}^{\prime}, \mathbf{u}_{g}$, and $\mathbf{u}_{g}^{\prime}$ are channel dependent. We keep track of the channel indices $\gamma$ and $\gamma^{\prime}$ whenever necessary to avoid confusion.
[31] B. C. Sawyer, B. L. Lev, E. R. Hudson, B. K. Stuhl, M. Lara, J. L. Bohn, and J. Ye, Phys. Rev. Lett. 98, 253002 (2007).

[32] H.-S. Lee, A. B. McCoy, R. R. Toczyłowski, and S. M. Cybulski, J. Chem. Phys. 113, 5736 (2000).

[33] J. G. E. Harris, R. A. Michniak, S. V. Nguyen, N. Brahms, W. Ketterle, and J. M. Doyle, Europhys. Lett. 67, 198 (2004). 